\title{
Serum titres of Pneumocystis carinii antibody in health care workers caring for patients with AIDS
}

\author{
T R Leigh, M J Millett, B Jameson, J V Collins
}

\begin{abstract}
Background-Pneumocystis carinii pneumonia was thought to occur from reactivation of latent infection, but recent studies with the polymerase chain reaction have failed to detect $P$ carinii in normal subjects. If pneumocystis pneumonia is therefore caused by new infection the source and mode of transmission of $P$ carinii remains unknown.

Methods-Natural exposure to $P$ carinii was detected by measuring antibodies by indirect immunofluorescence in 24 health care workers working continuously with patients with AIDS and 24 control health care workers exclusively treating elderly patients.

Results-P carinii antibody titres were significantly higher in the health care workers exposed to AIDS than in the control group (median titre 1:32 $v$ 1:16 respectively). Three control subjects had no antibodies compared with none of the subjects exposed to AIDS, and 10 of the 12 highest titres came from the exposed group.

Conclusions-Raised $\boldsymbol{P}$ carinii antibody titres in health care workers caring for patients with AIDS suggest that patients infected with HIV may be a potentially infectious source of $P$ carinii for susceptible subjects.
\end{abstract}

(Thorax 1993;48:619-621)

Department of

Respiratory Medicine, Chelsea and

Westminister

Hospital, London

SW10 9NH

T R Leigh

Department of Microbiology, Royal Marsden Hospital, London SW3 6JJ M J Millett

B Jameson

Royal Brompton

National Heart

Hospital, London

SW3 6NP

J V Collins

Correspondence to:

DrT R Leigh

Received 6 November 1992 Returned to authors 26 January 1993 Revised version received 10 February 1993 Accepted 16 February 1993 infection after immunosuppression 8 reactivation hypothesis has recently been questioned by several studies that have failed to detect latent $P$ carinii infection in normal subjects despite the use of highly sensitive techniques using the polymerase chain reaction..$^{10}$ Pneumocystis pneumonia may therefore develop from a new infection with $P$ carinii rather than from latent infection. This has raised questions about possible sources of $P$ carinii and, with recent case reports of pneumocystis pneumonia occurring after documented exposure, ${ }^{11} 12$ suggests that subjects with active pneumocystis pneumonia may be potentially infectious. Although airborne $P$ carinii causes pneumonia in rats, ${ }^{13}$ the same has not been shown to occur in humans. If, like rats, humans become infected with $P$ carinii by this route high antibody titres would be expected in immunocompetent subjects working closely with patients with AIDS, who are known carriers of the organism. ${ }^{14}$ We therefore compared $P$ carinii antibody titres in health care workers working with patients with AIDS and in a control group of health care workers.

\section{Methods}

Two groups of 24 health care workers were studied; all subjects lived and worked in London. The first group (17 women, seven men, mean age 33 years) had worked exclusively with patients with AIDS or those seropositive for HIV for at least six months before the study (mean duration 3.2 years) in a unit where about 200 new cases of pneumocystis pneumonia are diagnosed annually. The second, control, group (23 women, one man, mean age 42 years) had worked exclusively in dedicated geriatric hospitals and had never knowingly cared for patients positive for HIV or worked in a hospital treating patients with AIDS. All subjects were healthy and receiving no immunosuppressive drugs. Apart from two homosexuals in the exposed group who were negative for HIV in serial tests, all subjects were at low risk of HIV infection. Specimens of serum were taken from all subjects and saved at $-80^{\circ} \mathrm{C}$ for antibody testing.

DETECTION OF ANTIBODIES

All specimens were randomised and coded to ensure blind testing for antibodies. $P$ carinii antibodies were detected by indirect immunofluorescence using sections of human postmortem lung which were heavily infected with $P$ carinii. These sections had been fixed in Bouin's fluid to destroy existing immunoglobulin and preserve the pneumo-




cystis polysaccharide antigen. Sections were deparaffinised, rehydrated, and washed for 30 minutes in phosphate buffered saline at $\mathrm{pH}$ $7 \cdot 4$. The slides were then reacted for $30 \mathrm{~min}$ utes with a sample of each subject's serum diluted 1:8 with phosphate buffered saline. After washing, the slides were treated for 30 minutes with a 1:32 dilution of sheep derived fluorescein labelled whole antihuman immunoglobulin (Murex Diagnostics, Dartford). After washing, slides were mounted under phosphate buffered saline/glycerol (Citifluor, Citifluor Ltd, London) to preserve fluorescence, and viewed at $50 \times$ magnification under oil immersion and ultra violet light. Positive and negative control serum samples were included to confirm adequate staining, and all slides were viewed independently by two observers. Positive specimens, identified by clumps of fluorescing pneumocysts, were tested further at increasing, doubling, serum dilutions-that is, $1: 16,1: 32$, etc-until a negative result was obtained, and specimens giving different results between the two observers were repeated. Specimens with titres of $1: 128$ or more were also tested for IgM specific $P$ carinii antibody with the same method but substituting IgM specific antihuman antibody for the whole antihuman immunoglobulin (Murex Diagnostic, Dartford). Results for the two groups were analysed, after breaking the code, with the Mann-Whitney test.

\section{Results}

There was good overall agreement between the two observers, with a concordance rate of $90 \%$. A high background fluorescence, the most frequent cause of discrepancies, was invariably resolved after restaining.

Overall, 45 of the 48 specimens (94\%) had

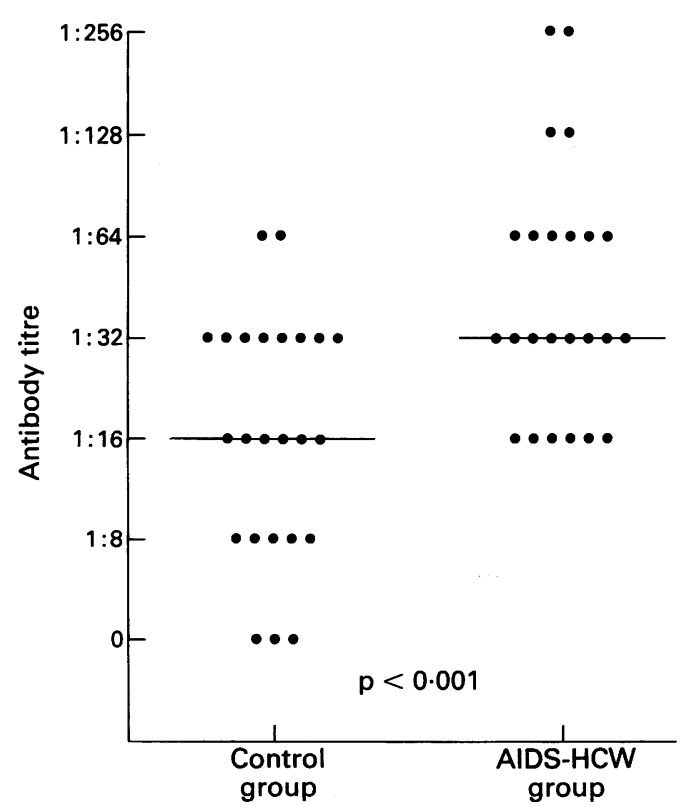

$P$ carinii total antibody titres with median values in health care workers caring for patients with AIDS (AIDS-HCW) and those caring for elderly patients (control group).
$P$ carinii IgM titres in four patients with total antibody titres of $1: 128$ and above

\begin{tabular}{lll}
\hline Case no & Total antibody titre & IgM titre \\
\hline 1 & $1: 256$ & $1: 8$ \\
2 & $1: 256$ & $1: 64$ \\
3 & $1: 128$ & - \\
4 & $1: 128$ & - \\
\hline
\end{tabular}

detectable antibody to $P$ carinii at dilutions of $1: 8$ and above (fig). The three specimens negative for antibody all came from the control group, whereas the four samples with titres of $1: 128$ and above (which were also tested for $P$ carinii IgM) came from the group exposed to AIDS. Antibody titres in the exposed group were significantly higher than in the control group, with a median titre of $1: 32$ (range $1: 16$ to $1: 256) v 1: 16$ (0 to $1: 64$ ), respectively $(\mathrm{p}<0.001)$. IgM was not detectable in samples from the two subjects with total antibody titres of $1: 128$, and $\operatorname{IgM}$ titres of 1:8 and 1:64 were found in the samples from the other two subjects with total antibody titres of 1:256 (table). Neither of these latter two subjects had recently had respiratory illness and both remained symptom free. One (case 1) was a nurse responsible for helping with inpatient bronchoscopies to investigate patients with suspected pneumocystis pneumonia, and the other (case 2) was a research nurse who had been working with patients with AIDS for seven years. We found no relation between antibody titre and length of time working with patients infected with HIV or between the antibody titres in medical and nursing staff.

\section{Discussion}

The finding of an overall high prevalence of $P$ carinii antibodies agrees with most other seroprevalence studies. The wide variation in the prevalence detected in these studies, ranging from $8.8 \%$ of blood donors, ${ }^{4} 57 \%$ of normal controls, ${ }^{5}$ and $85 \%$ of normal adults, ${ }^{67}$ to $100 \%$ of two year old children, ${ }^{15}$ may be partly explained by different laboratory techniques but may also reflect geographical or seasonal differences in the prevalence of $P$ carinii. Although animal studies have clearly shown the infectious nature of $P$ carinii, ${ }^{13}$ recommendations to isolate infected individuals $^{16}$ have been largely ignored in the belief that the population is already latently infected with $P$ carinii. With mounting evidence refuting this hypothesis of latent infection, ${ }^{910}$ the potentially infectious nature of $P$ carinii has become more important.

Our results support the hypothesis that $P$ carinii may be potentially infectious by showing raised antibody titres and therefore exposure to $P$ carinii in health care workers caring for patients with AIDS compared with control health care workers. Although we could not completely exclude the possibility of covert infection with HIV without resorting to serial HIV tests, our selection criteria kept this possibility to a minimum. Differences in the age and sex distribution of 
the two groups were small and probably did not skew the difference in antibody titres between the two groups. Another possible source of error was the two homosexuals in the exposed group, who may have been exposed to $P$ carinii socially rather than professionally. Both their antibody titres, however, were below the group average at 1:16, and they had no haematological evidence of immune paresis.

The presence of significant IgM titres in association with the highest total antibody titres may indicate that these individuals had been recently exposed to $P$ carinii, although both subjects remained free of respiratory symptoms. The high antibody titres of the bronchoscopy nurse are noteworthy because she was probably being regularly exposed to fluid aerosols containing $P$ carinii. Although we cannot eliminate other, unidentified, sources of $P$ carinii as the cause of raised antibody titres, these findings suggest that patients with AIDS may be a potentially infectious source of $P$ carinii to susceptible individuals. As different serotypes of human $P$ carinii can now be distinguished by molecular biological techniques, some of the questions about the epidemiology and transmission of $P$ carinii infection may soon be resolved.

In conclusion, this study adds to the growing evidence that infection with $P$ carinii may be transmitted from infected carriers, who may be a potential source of pneumocystis pneumonia in immunosuppressed subjects.

We thank Dr David Warhurst of the London School of Hygiene and Tropical Medicine for supplying the fixed blocks of human postmortem lung. TRL is supported by a grant from North West Thames Regional Health Authority.

1 Murray JF, Mills J. Pulmonary infectious complications of human immunodeficiency virus infection. Part 2. Am Rev Respir Dis 1990;141:1582-98.
2 Bennett CL, Gertler P, Guze PA, Gartinkle JB, Kanouse DE, Greenfield S. The relation between resource use and in-hospital mortality for patients with acquired immunodeficiency syndrome-related Pneumocystis carinii pneumonia. Arch Intern Med 1990;150:1447-52.

3 Pifer LL, Hughes WT, Stagno S, Woods D. Pneumocystis carinii infection: evidence for high prevalence in normal and immunosuppressed individuals. Pediatrics 1978; 61:35-41.

4 Chatterton JMW, Joss AWL, Williams H, Ho-Yen DO. Pneumocystis carinii antibody testing. $f$ Clin Pathol 1989;42:865-8.

5 Shepherd V, Jameson B, Knowles GK. Pneumocystis carinii pneumonitis: a serological study. $\mathcal{f}$ Clin Pathol 1979;32:773-7.

6 Kovacs JA, Halpern JL, Swan JC, Moss J, Parrillo JE, Masur $\mathrm{H}$. Identification of antigens and antibodies specific for Pneumocystis carinii. F Immunol 1988;140: 2023-31.

7 Peglow SL, Smulian GA, Linke MJ, Pogue CL, Nurre S, Crisler J, et al. Serologic responses to Pneumocystis carinii antigens in health and disease. F Infect Dis 1990;161:296-306.

8 Masur H, Lane HC, Kovacs JA, Allegra CJ, Edman JC. Pneumocystis pneumonia: from bench to clinic. Ann Intern Med 1989;111:813-26.

9 Peters SE, Wakefield AE, Sinclair K, Millard PR, Hopkin JM. A search for $P$ carinii in post-mortem lungs by DNA amplification. F Pathol 1992;166:195-8.

10 Leigh TR, Wakefield AE, Peters SE, Hopkin JM, Collins JV. Comparison of DNA amplification and immunofluorescence for detecting Pneumocystis carinii in patients receiving immunosuppressive therapy. Transplantation 1992;54:468-70.

11 Chave J-P, David S, Wauters J-P, Glauser MP, Francioli $P$. Is Pneumocystis carinii transmissable from AIDS to other patients? A case-control study of a cluster of PC pneumonia in renal transplant recipients. Proceedings of the sixth international conference on AIDS. San Francisco. 1990. Vol 1. Th.B.392.220.

12 Goesch TR, Götz G, Stellbrinck KH, Albrecht H, Weh HJ, Hossfeld DK. Possible transfer of Pneumocystis carinii between immunodeficient patients. Lancet 1990; 336:627.

13 Hughes WT. Natural mode of acquisition for de novo infection with Pneumocystis carinii. F Infect Dis 1982; 145:842-8.

14 Leigh TR, Gazzard BG, Kangro HO, Jeffries DJ, Collins JV. DNA amplification by the polymerase chain reaction to detect sub-clinical Pneumocystis carinii colonisation in HIV positive individuals with and without respiratory symptoms. Respir Med (in press).

15 Meuwissen JHET, Tauber I, Leewenberg ADEM, Beckers PJA, Sieben M. Parasitologic and serologic observations of infection with pneumocystis in humans. F Infect Dis 1977;136:43-9.

16 Singer C, Armstrong D, Rosen PP, Schottenfeld D. Pneumocystis carinii pneumonia: a cluster of eleven cases. Ann Intern Med 1975;82:772-7. 